\title{
Photographie aérienne et appréciation de l'évolution du dépérissement de l'épicéa en Ardennes
}

\author{
C. Farcy
}

Unité des Eaux et Forêts, Place Croix-du-Sud, 2 (bte 4), Université catholique de Louvain, B-1348 Louvain-la-Neuve, Belgique

(reçu le 20-1-1988, accepté le 5-5-1988)

Résumé - Sur la base de photographies aériennes infrarouge couleurs au $1 / 5000$ prises à 2 années d'intervalie (1983 et 1985), 600 épicéas répartis dans un réseau préalablement stratifié (âge, état sanitaire général) de 60 placettes permanentes ont été interprétés en vue de déterminer un schéma évolutif des symptômes de dépérissement qui les affectent. L'état sanitaire de $70 \%$ des arbres est resté stable; celui des $30 \%$ restants s'améliore ou se dégrade dans des sens divers avec une tendance privilégiée vers le stade chlorose. Pour que les critères de stratification puissent s'exprimer, il faudrait vraisemblablement tenir compte des facteurs stationnels.

chlorose - dépérissement - épicéa - évolution des symptômes - photographie aérienne placette permanente

Summary - Aerial photography and valuation of Norway Spruce decline evolution in the Ardennes. A diagram of the decline symptoms evolution has been carried out using infrared color aerial photographies (scale 1/5 000) taken in 1983 and 1985 : after a stratification of Norway Spruce stands (age, 2 levels; general healt state, 3 levels), 10 permanent plots including 10 trees have been identified in each of the 6 strata. The health state of $70 \%$ of the trees doesn't change. The other $30 \%$ improve or degrade following different ways with a privileged propensity to the chlorosis stage. A study taking into account ecological factors would permit a beter analysis of stratification criteria.

chlorosis - decline - Norway Spruce - evolution of symptoms - aerial photography - permanent plot

\section{Introduction}

Avant les années 80 , l'état sanitaire des peuplements ne posait, en général, pas de problèmes majeurs au forestier. Une description précise des couronnes saines n'existait pas. Seuls quelques phénotypes typiques ou symptômes d'attaque parasitaire classique sont décrits dans des ouvrages de sylviculture.

A la suite des événements qui se sont produits dans certaines forêts ardennaises dans le courant de l'hiver 1982-1983, provoquant l'inquiétude de maints forestiers 
- des épicéas des Hautes-Fagnes (Eupen) ont perdu brutalement d'importantes quantités d'aiguilles - , les chercheurs belges, après leurs collègues allemands, se sont penchés sur le problème et ont constaté des troubles partiellement inexpliqués (Weissen, 1983), rappelant ceux du Tannensterben rapportés par la littérature allemande (Weissen et al., 1984).

La recherche d'une méthode permettant d'estimer l'étendue des dégâts s'est rapidement révélée primordiale. Les difficultés majeures liées à la réalisation d'inventaires sanitaires au sol ont plaidé en faveur des techniques de télédétection.

L'épicéa occupe environ un tiers de la forêt belge et joue un rôle non négligeable dans l'économie de ce pays. II a été étudié de façon privilégiée, notamment par l'Uni- té des Eaux et Forêts de l'UCL qui, en collaboration avec la société Walphot SA, a mis au point une méthode d'inventaire des pessières ardennaises. La procédure est basée sur un sondage systématique sur photographies aériennes infrarouge couleurs (IR-C) d'échelle 1/5000 : une grille de points à maille carrée de $8 \mathrm{~mm}$ de côté (40 m au sol) est placée sur le cliché; les 5 couronnes les plus proches de chaque point (taux de sondage moyen de 31,25 arbres/ha) sont examinées et affectées à une des 7 classes sanitaires préalablement définies sur la base d'une notion d'intensité des dégâts (Giot et al., 1985).

Eprouvée comme technique d'inventaire, la méthode est cependant mal adaptée à l'étude de l'évolution des dommages dans le temps : en comparant globalement les résultats d'inventaires annuels

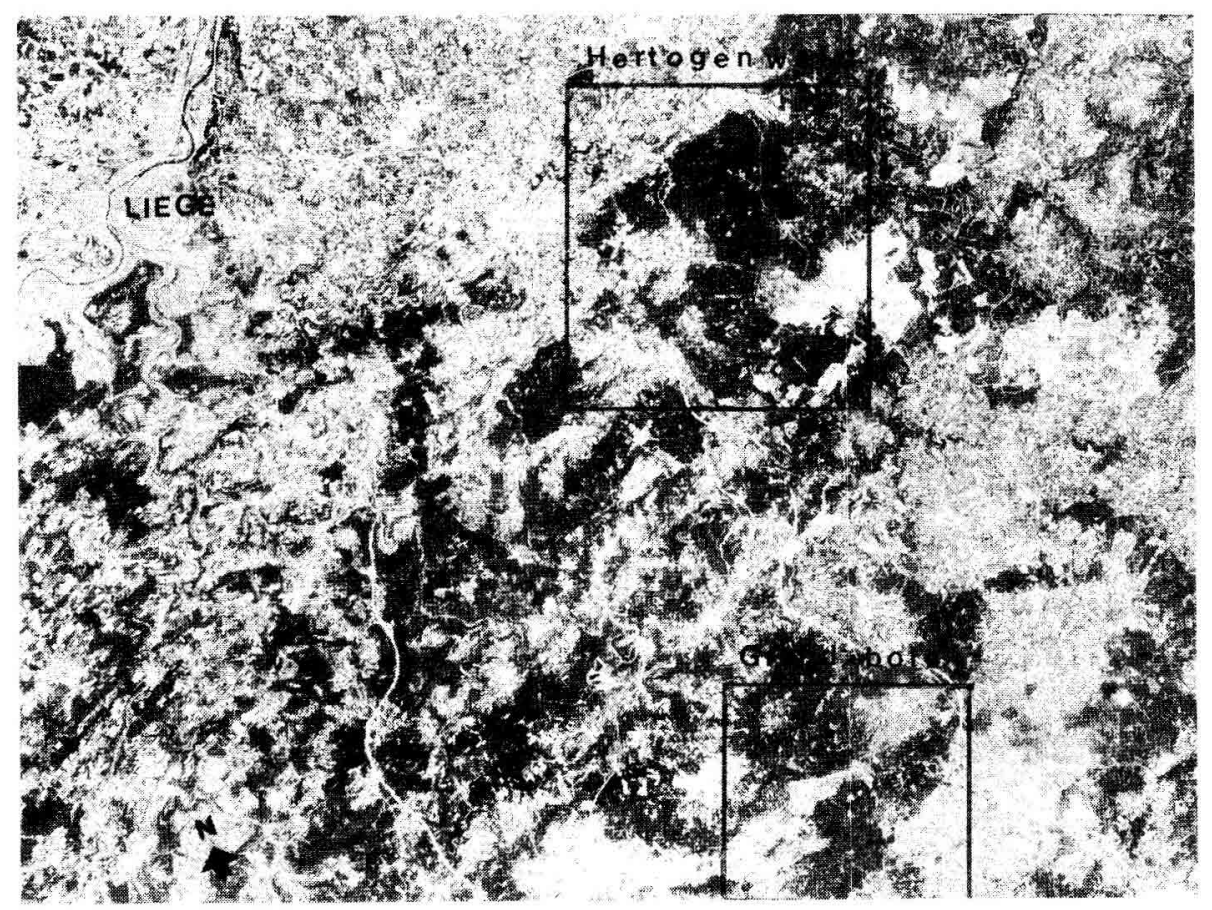

Fig. 1. Localisation des zones test sur une image de Thematic Mapper. 


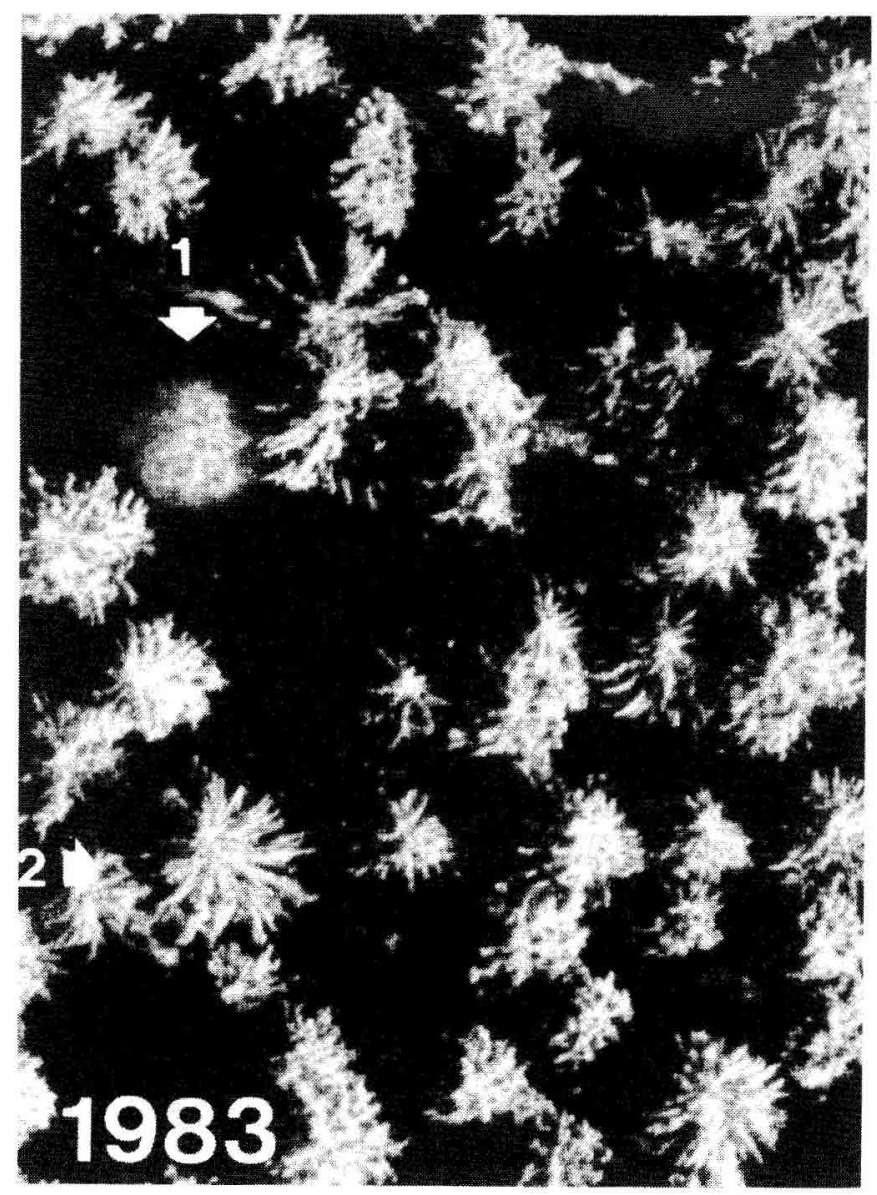

Fig. 2. Agrandissement (1/500) d'une diapositive IR-C de 1983.

successifs (quelque 6000 arbres), on n'observe pas d'évolution cohérente. II est probable que le taux d'arbres passant d'une classe sanitaire à une autre est inférieur au coefficient de variation relatif à l'estimation des effectifs de chaque classe, ou encore, que l'évolution se fait dans des sens divers la rendant indétectable par cette voie.

La recherche d'une méthode d'analyse de la dynamique du dépérissement de l'épicéa sur photographie aérienne IR-C constitue l'objectif principal de la présente étude dont une des phases consiste en l'élaboration de nouvelles définitions des classes sanitaires sur la base d'une notion de différenciation des divers symptômes.

\section{Zones test et protocole}

Deux zones test ont été retenues, le GrandBois de Vielsalm et l'Hertogenwald (Fig. 1). Ces deux massifs se situent dans une région où d'importants dégâts avaient été constatés pendant l'hiver 1982-1983. 


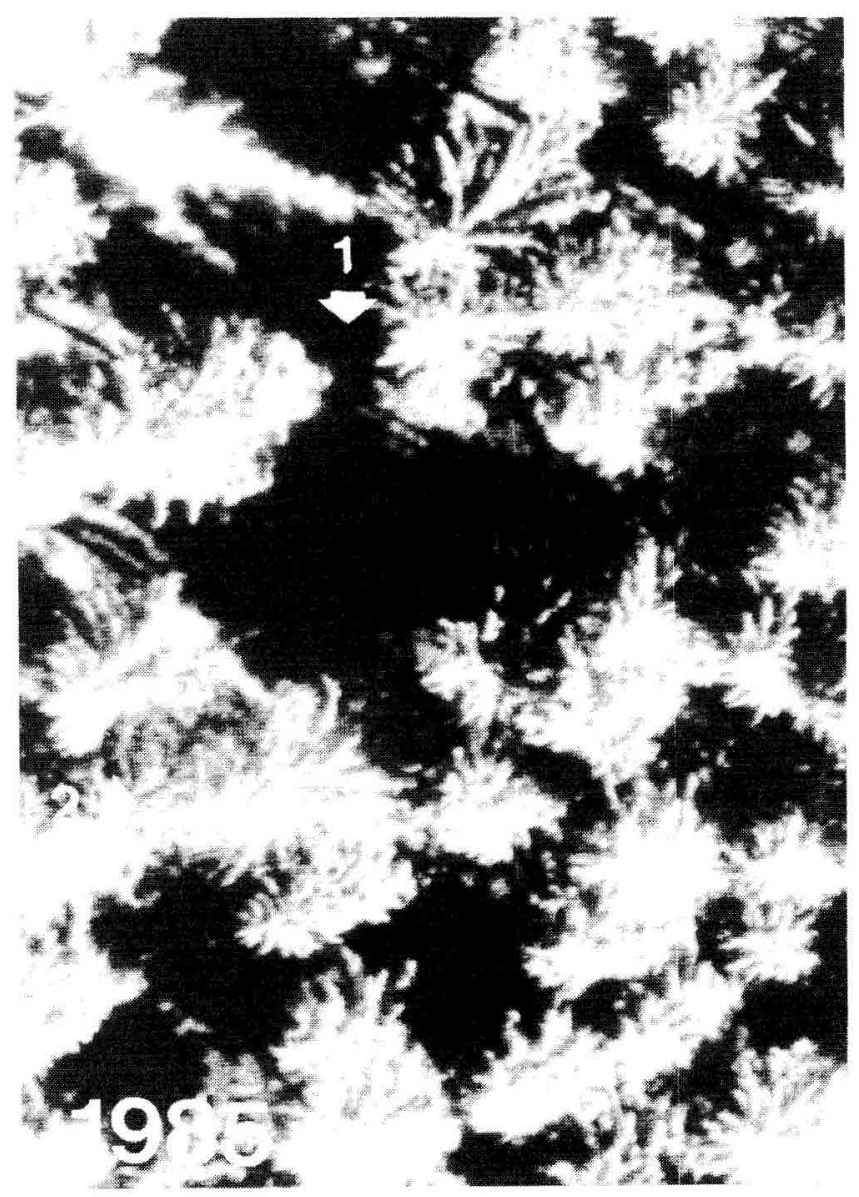

Fig. 3. Agrandissement (1/500) d'une diapositive IR-C de 1985

Le choix de la méthodologie résulte des caractéristiques intrinsèques de la photographie aérienne : c'est un document d'archive qui constitue une mémorisation du paysage à un moment donné qu'aucune description de terrain ne peut égaler. Depuis 1983, la couverture aérienne complète de nos zones test est réalisée annuellement (diapositive IR-C verticale, 23 $x 23 \mathrm{~cm}, 1 / 5000$, focale $15 \mathrm{~cm}$ ). Ces documents permettent de procéder à une observation des symptômes affectant une même couronne d'épicéa à 2 années d'intervalle, laps de temps choisi pour cette première approche méthodologique. Un exemple est illustré par les Figures 2 et 3 : l'arbre 1 qui était sec en 1983 a disparu en 1985 , vraisemblablement suite à une éclaircie; l'arbre 2 affecté de quelques points de chlorose en 1983 est retrouvé mort 2 ans plus tard.

Après une stratification des diapositives de 1983, des placettes de 10 arbres sont choisies sur ces clichés et ses individus sont positionnés sur ceux de 1985. Ensuite, chaque couronne est classée sanitairement d'après les documents de 1983 et d'après ceux de 1985. Le détail de ces différentes étapes est donné cidessous sans respecter toutefois la chronologie du travail effectué; en effet la définition des 
classes sanitaires est un préalable nécessaire à sa bonne compréhension.

\section{Classification sanitaire}

L'affectation à une classe sanitaire est déterminée par la présence d'un des symptômes détectables sur diapositive IR-C 1/5000 par des critères de teinte et de structure de cime. Les symptômes ont été ordonnés a priori en imaginant une séquence allant de la chlorose à la mort, en passant par la perte d'aiguilles; cette hypothèse reste à vérifier.
Le Tableau I détaille le nouvel agencement des classes et le compare à celui qui est utilisé lors des inventaires sanitaires de pessières par la méthode de sondage systématique précitée. La structure "en port drapé" est sujette à controverse : ce port est fréquent chez les provenances de basse altitude (Schmidt-Vogt, 1977) mais certains l'interprètent comme symptôme de dépérissement; c'est pour cette raison qu'il occupe une place marginale dans notre classement.

La présence d'un symptôme est déterminante pour l'appartenance à une classe mais n'exclut pas l'existence des symptômes précédents

Tableau I. Classement des symptômes de dépérissement affectant l'épicéa et image homologue de ceux-ci sur diapositive IR-C verticale d'échelle $1 / 5000$.

\begin{tabular}{|c|c|c|c|}
\hline $\begin{array}{l}\text { Classe sanitaire } \\
\text { pour l'étude de l' } \\
\text { évolution des } \\
\text { symptômes }\end{array}$ & $\begin{array}{l}\text { Classe sanitaire } \\
\text { pour l'inventaire } \\
\text { sanitaire }\end{array}$ & $\begin{array}{l}\text { Symptôme sur } \\
\text { le terrain }\end{array}$ & $\begin{array}{l}\text { Image homologue } \\
\text { des symptômes sur } \\
\text { diapositive IR-C } \\
\text { 1/5000 }\end{array}$ \\
\hline 0 & 0 & $\begin{array}{l}\text { Sain sans } \\
\text { port drapé }\end{array}$ & $\begin{array}{l}\text { Cime touffue de teinte } \\
\text { magenta }\end{array}$ \\
\hline $1^{\circ}$ & 1 & Port drapé & $\begin{array}{l}\text { Cime étoilée de teinte } \\
\text { magenta }\end{array}$ \\
\hline $1^{\prime}$ & & $\begin{array}{l}\text { Chlorose } \\
\text { légère }\end{array}$ & $\begin{array}{l}\text { Taches blanc brillant sur } \\
\text { fond magenta }\end{array}$ \\
\hline $2^{\prime}$ & 2 & $\begin{array}{l}\text { Chlorose } \\
\text { généralisée }\end{array}$ & $\begin{array}{l}\text { Teinte blanc brillant } \\
\text { gagnant toute la cime }\end{array}$ \\
\hline $2^{\prime \prime}$ & & $\begin{array}{l}\text { Perte d'aiguilles } \\
\text { légère (sur toute } \\
\text { la hauteur sauf la } \\
\text { flèche terminale) }\end{array}$ & $\begin{array}{l}\text { Vision des branches de } \\
\text { teinte cyan et/ou teinte } \\
\text { gris vert sauf au sommet }\end{array}$ \\
\hline \multirow[t]{5}{*}{+} & 3 & $\begin{array}{l}\text { Perte d'aiguilles } \\
\text { généralisée (sur } \\
\text { toute la hauteur) }\end{array}$ & $\begin{array}{l}\text { Teinte gris vert gagnant } \\
\text { le sommet }\end{array}$ \\
\hline & 4 & $\begin{array}{l}\text { Mort de la flèche } \\
\text { terminale }\end{array}$ & Sommet de teinte cyan \\
\hline & 5 & Mort & Teinte cyan généralisée \\
\hline & 6 & Douteux & Douteux \\
\hline & 7 & Chablis & $\begin{array}{l}\text { Section circulaire de } \\
\text { teinte cyan au centre } \\
\text { de ia cime. }\end{array}$ \\
\hline
\end{tabular}


dans le classement. La classe 1' par exemple, regroupe des individus affectés de chlorose légère avec ou sans port drapé.

\section{Stratification}

Les hypothèses émises sur les causes du dépérissement sont nombreuses (Niesslein et Voss, 1985). L'attitude de Rehfuess (1985) est révélatrice à cet égard : “... La variabilité de manifestation du mal indique qu'il s'agirait d'un complexe de types de dommages divers, induits par un ensemble de causes différentes. L'apparition presque simultanée de l'ensemble des dégâts laisse supposer qu'il doit exister un facteur de synchronisation. Comme les zones polluées ou non sont également touchées, la nature de ce facteur serait climatique..."

Toutefois, il semble qu'une des hypothèses actuellement avancée et relative à l'influence des conditions stationnelles en relation directe avec la pauvreté originelle du sol, soit à approfondir (Weissen, 1983; Hüttl, 1985; Tan et al., 1986).

Les inventaires sanitaires par sondage systématique effectués dans nos zones test ont montré l'existence d'une relation directement proportionnelle entre l'âge et l'état sanitaire (Aboussouan, 1985; Giot et al., 1985). Qu'en est-il de la dynamique de cet état sanitaire ? Des symptômes identiques affectant des arbres d'âge différent, évolueront-ils de la même façon?

Par ailleurs, en examinant la répartition spatiale des dégâts, on n'observe pas d'effet de contagion : on rencontre, dans la plupart des cas, les divers types de symptômes à des degrés d'intensité variables, réunis au sein d'un espace restreint. On peut néanmoins se demander si l'évolution de l'état sanitaire d'un arbre individuel est influencée par l'état sanitaire général du peuplement auquel il appartient?

Pour tenter de répondre à ces deux questions, nous avons procédé à une stratification des pessières sur les clichés de 1983, d'après l'âge et l'état sanitaire général :

L'âge. Deux niveaux : 50 à 79 ans, 80 ans et plus. Il a été fourni par la connaissance des parcelles photographiées. Les peuplements d'âge inférieur à 50 ans n'ont pas été considérés en raison des difficultés de repérage de leurs couronnes sur les photos d'une année et de transfert sur les documents d'une autre mission aérienne. Ces difficultés résultent de l'imbrication des couronnes due au nombre élevé de pieds par hectare et de l'absence, sur l'image photographique, de dessins caractéristiques ou de signes particuliers du toit de la végétation pouvant servir de points de repère. Notons à ce sujet l'utilité d'un restituteur numérique; il en sera question par la suite.

L'état sanitaire général. Trois niveaux : 0 à $9 \%$ de classe $2 ; 10$ à $19 \%$ de classe $2 ; 20 \%$ et plus de classe 2. Disposant des résultats de l'inventaire sanitaire par sondage systématique des peuplements de nos zones test, réalisé sur les photographies aériennes de 1983, nous avons défini “'état sanitaire général d'un peuplement" sur la base des fréquences observées de la classe 2 (Tableau I). Le choix de cette classe résulte de la bonne représentativité numérique de ses effectifs ce qui n'est pas le cas des classes 3,4 ou 5 . D'autre part, elle regroupe des individus affectés de symptômes indicateurs d'un stade avancé du mal et caractérisant, mieux que la classe 1 , un état sanitaire général déficient.

\section{Application}

Au sein de chacune des 6 strates ainsi obtenues sur les diapositives IR-C 1/5000 de 1983 , 10 placettes de 10 arbres ont été choisies. Compte tenu des caractéristiques géométriques de la photographie aérienne verticale (déversement des arbres en bordure de photo...) nous n'avons travaillé que dans la zone centrale de celle-ci (Remeijn, 1981). L'étape suivante a été le repérage précis des 600 couronnes sur les clichés de 1985. C'est pour faciliter ce travail que l'étude d'arbres groupés dans des placettes a été préférée à celle d'individus isolés; en effet, un groupe d'arbres est plus aisément identifiable et transférable sur les clichés de missions aériennes différentes.

Le choix imposé de l'échelle n'a pas facilité la recherche des couronnes homologues en parti- 
culier quand il s'agissait de peuplements très denses; quelquefois, nous avons donc eu recours à des points de repère (bord de lisière, couronne remarquable, ...). Dans ces cas-là, les placettes n'ont pas été choisies de façon parfaitement aléatoire; nous resterons donc prudents et ne tenterons pas de généraliser les informations recueillies à toute la zone.

A propos de la récolte des données, il s'est avéré intéressant de procéder par observation pairée : une couronne est examinée sur les diapositives de 1983 et son état sanitaire est comparé à celui de 1985. En ayant sous les yeux simultanément les deux images, on élimine pour une bonne part la subjectivité de l'interprète qu'il faut déplorer lors d'une observation isolée (Delincé, 1986). Dans le même ordre d'idées, la variation de teinte des photographies d'une mission à l'autre ne pose pas de problèmes majeurs.

\section{Aspect statistique}

L'étude de l'évolution d'un phénomène par dénombrement d'individus classés dans différentes catégories, relève de l'analyse des données discrètes. Le traitement statistique classique qui lui est appliqué est basé sur l'analyse des tables de contingence (Bishop et al., 1975). Notre échantillonnage étant stratifié létats sanitaires en 1983 et en 1985, âge, état sanitaire général, placette) nous aurions pu faire appel à l'arsenal statistique disponible pour l'analyse des tables de contingence à plusieurs critères. Le temps dont nous disposions et le souci de répondre avant toute chose à l'objectif principal de cette étude (analyse des potentialités de la photographie aérienne) nous ont amenés à procéder, en première analyse, de façon simplifiée.

Dans une premier temps, nous avons considéré la population dans son ensemble $(600$ individus) et la répartition de ses effectifs d'après 2 critères : état sanitaire en 1983, état sanitaire en 1985, chacun d'eux distinguant 6 niveaux (Tableau 1). Nous avons consigné les individus dans une table de contingence à 2 entrées et y avons testé l'indépendance, la symétrie, la symétrie relative et l'homogénéité marginale (Bishop et al., 1975). Ensuite, nous avons examiné, pour les individus appartenant à une classe sanitaire donnée en 1983, les fréquences observées de transition de classe.

Dans un second temps, nous avons divisé la population en autant de sous-populations qu'il y a de niveaux par critères et par combinaisons entre critères et avons consigné leurs effectifs en autant de tables de contingence à deux entrées (états sanitaires en 1983 et en 1985). Disposant ainsi de sous-populations qui devraient être plus homogènes, nous y avons testé les mêmes hypothèses que pour la population considérée dans son ensemble. La comparaison des résultats tests nous permettra soit de dégager d'éventuelles interactions masquées par le regroupement des sous-populations en une seule table, soit de conclure sur l'opportunité du choix des caractéristiques ayant servi à la stratification.

Une remarque s'impose au sujet du critère "placette": chacune d'elles n'étant composée que de 10 individus, il serait imprudent de leur appliquer les tests d'hypothèses précités car ils se révèleraient trop peu puissants (Everitt, 1977); nous traiterons donc les données dans leur ensemble sans considérer l'effet placette.

\section{Résultats}

Pour la population prise dans son ensemble, il y a rejet de l'hypothèse d'indépendance entre les états sanitaires en 1983 et $1985(\alpha=0,01)$. II y a donc association entre ces 2 critères. L'hypothèse de symétrie est également rejetée. En d'autres mots, il semble qu'il y ait eu un sens privilégié d'évolution de l'état sanitaire entre 1983 et 1985 . Le rejet de I'hypothèse de symétrie relative prouve que cette évolution qui aurait pu être apparente est bien réelle et que ne peut être mise en cause une différence entre les effectifs des classes en 1983. Le rejet de l'hypothèse d'homogénéité des marges confirme ces premières constatations.

Le rejet des différentes hypothèses, observé pour la population prise dans son 
ensemble, se répète de façon systématique si l'on considère séparément les diverses sous-populations. Autrement dit, quelles que soient les classes d'âge et d'état sanitaire général d'un peuplement, d'une part, il y a association entre l'état sanitaire d'un arbre en 1983 et en 1985 et, d'autre part, l'évolution se manifeste plus sensiblement dans un sens que dans l'autre.

L'analyse des fréquences observées de transition montre s'il s'agit d'une évolution qui mène à la dégradation ou à l'amélioration; en d'autres termes, si un arbre passe d'une classe à une autre entre 1983 et 1985 , est-ce vers une classe plus saine ou plus dégradée ? (Tableau II).

Les classes 2' et + étant très faiblement représentées dans l'échantillon (respectivement 11 et 15 arbres en 1983), nous ne pouvons considérer leurs valeurs comme significatives. Dans le même ordre d'idées, il serait imprudent d'attacher trop d'importance aux résultats concernant les

Tableau II. Fréquence observée de transition après deux ans (en \% des effectifs des classes sanitaires de 1983)

\begin{tabular}{|c|c|c|c|c|}
\hline & $\begin{array}{l}\text { Classe } \\
\text { en } 1983\end{array}$ & \multicolumn{2}{|c|}{ Fréquence observée de transition } & Amélioration \\
\hline \multirow{6}{*}{$\begin{array}{l}\text { A. Tous âges et } \\
\text { états sanitaires } \\
\text { confondus } \\
\text { (effectif } 600 \text { ) }\end{array}$} & $\mathrm{Cl} .0$ & 71,0 & 29,0 & $-\left({ }^{*}\right)$ \\
\hline & $\mathrm{Cl} .1^{\circ}$ & 64,1 & 35,9 & - \\
\hline & Cl.1' & 66,1 & 31,2 & 2,7 \\
\hline & Cl.2' & 81,8 & - & 18,2 \\
\hline & $\mathrm{Cl} .2^{\prime \prime}$ & 65,8 & 5,5 & 28,7 \\
\hline & Cl.t & 53,3 & - & 46,7 \\
\hline \multirow{6}{*}{$\begin{array}{l}\text { B. } 50 \text { à } 79 \text { ans } \\
\text { (effectif } 300)\end{array}$} & Cl.0 & 72,0 & 28,0 & - \\
\hline & $\mathrm{Cl} .1^{\circ}$ & 68,7 & 31,3 & - \\
\hline & Cl.1' & 56,0 & 42,0 & 2,0 \\
\hline & Cl.2' & 85,7 & - & 14,3 \\
\hline & $\mathrm{Cl} .2^{\prime \prime}$ & 72,0 & 8,5 & 19,1 \\
\hline & Cl.t & 100,0 & - & - \\
\hline \multirow{6}{*}{$\begin{array}{l}\text { C. }>=80 \text { ans } \\
\text { (effectif } 300 \text { ) }\end{array}$} & $\mathrm{Cl} .0$ & 69,0 & 31,0 & - \\
\hline & $\mathrm{Cl} .1^{\circ}$ & 57,8 & 42,2 & - \\
\hline & $\mathrm{Cl} .1^{*}$ & 74,6 & 22,0 & 3,4 \\
\hline & $\mathrm{Cl} .2^{\prime \prime}$ & 75,0 & - & 25,0 \\
\hline & $\mathrm{Cl} .2^{\prime \prime}$ & 62,6 & 4,0 & 33,4 \\
\hline & Cl.t & 46,1 & - & 53,9 \\
\hline \multirow{6}{*}{$\begin{array}{l}\text { D. } 0 \text { à } 9 \% \text { de } \mathrm{Cl} .2 \\
\text { (effectif } 200 \text { ) }\end{array}$} & $\mathrm{Cl} .0$ & 69,0 & 31,0 & - \\
\hline & $\mathrm{Cl} .1^{\circ}$ & 65,6 & 34,4 & - \\
\hline & Cl.1' & 64,3 & 33,3 & 2,4 \\
\hline & $\mathrm{Cl} .2^{\prime}$ & 75,0 & - & 25,0 \\
\hline & Cl.2" & 56,2 & 6,2 & 37,6 \\
\hline & Cl.t & 66,7 & - & 33,3 \\
\hline \multirow{6}{*}{$\begin{array}{l}\text { E. } 10 \text { a } 19 \% \\
\text { de } \mathrm{Cl} .2 \\
\text { (effectif 200) }\end{array}$} & Cl.o & 81,6 & 18,4 & - \\
\hline & $\mathrm{Cl} .1^{\circ}$ & 62,7 & 37,3 & - \\
\hline & Cl.1' & 70,4 & 27,3 & 2,3 \\
\hline & $\mathrm{Cl} .2^{\prime}$ & 100,0 & - & - \\
\hline & $\mathrm{Cl} .2^{\prime \prime}$ & 67,3 & 9,1 & 23,6 \\
\hline & $\mathrm{Cl} .+$ & 28,6 & - & 71,4 \\
\hline
\end{tabular}


Tableau II. (Suite)

\begin{tabular}{|c|c|c|c|c|}
\hline & $\begin{array}{l}\text { Classe } \\
\text { en } 1983\end{array}$ & $\begin{array}{l}\text { Fréquence ob } \\
\text { Stabilisation }\end{array}$ & $\begin{array}{l}\text { Ee de transition } \\
\text { Dégradation }\end{array}$ & Amélioration \\
\hline $\begin{array}{c}\text { F. }>=20 \% \text { de Cl. } 2 \\
\text { (effectif 200) }\end{array}$ & $\begin{array}{l}\text { Cl.0 } \\
\text { Cl. } 1^{\circ} \\
\text { Cl.1' } \\
\text { Cl.2" } \\
\text { Cl. } 2^{\prime \prime} \\
\text { Cl.t }\end{array}$ & $\begin{array}{l}63,6 \\
63,9 \\
60,9 \\
50,0 \\
74,4 \\
80,0\end{array}$ & $\begin{array}{r}36,4 \\
36,1 \\
34,8 \\
- \\
- \\
-\end{array}$ & $\begin{array}{r}- \\
- \\
4,3 \\
50,0 \\
25,6 \\
20,0\end{array}$ \\
\hline $\begin{array}{l}\text { G. } 50 \text { à } 79 \text { ans et } \\
0 \text { à } 9 \% \text { de } \mathrm{Cl} .2 \\
\text { (effectif 100) }\end{array}$ & 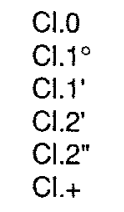 & $\begin{array}{r}79,3 \\
69,4 \\
57,1 \\
67,0 \\
60,0 \\
100,0\end{array}$ & $\begin{array}{r}20,7 \\
30,6 \\
42,9 \\
- \\
10,0 \\
-\end{array}$ & $\begin{array}{r}- \\
- \\
33,0 \\
30,0 \\
-\end{array}$ \\
\hline $\begin{array}{l}\text { H. } 50 \text { à } 79 \text { ans } \\
\text { et } 10 \text { à } 19 \% \\
\text { de } \mathrm{Cl} .2 \\
\text { (effectif } 100)\end{array}$ & $\begin{array}{l}\text { Cl.o } \\
\text { Cl. } 1^{\circ} \\
\text { Cl. } 1^{\prime} \\
\text { Cl. } 2^{\prime} \\
\text { Cl. } 2^{\prime \prime} \\
\text { Cl. }\end{array}$ & $\begin{array}{r}84,0 \\
61,8 \\
64,7 \\
100,0 \\
73,7 \\
100,0\end{array}$ & $\begin{array}{r}16,0 \\
38,2 \\
35,3 \\
15,8 \\
-\end{array}$ & $\begin{array}{r}- \\
- \\
- \\
10,5 \\
-\end{array}$ \\
\hline $\begin{array}{l}\text { 1. } 50 \text { à } 79 \text { ans } \\
\text { et }>=20 \% \\
\text { de } \mathrm{Cl} .2 \\
\text { (effectif } 100 \text { ) }\end{array}$ & $\begin{array}{l}\mathrm{Cl} .0 \\
\mathrm{Cl} .1^{\circ} \\
\mathrm{Cl} .1^{\prime} \\
\mathrm{Cl} .2^{\prime} \\
\mathrm{Cl} .2^{\prime \prime} \\
\mathrm{Cl} .+\end{array}$ & $\begin{array}{r}53,6 \\
73,8 \\
41,7 \\
- \\
77,8 \\
-\end{array}$ & $\begin{array}{r}46,4 \\
26,2 \\
50,0 \\
- \\
- \\
-\end{array}$ & $\begin{array}{r}- \\
8,3 \\
- \\
22,2 \\
-\end{array}$ \\
\hline $\begin{array}{c}\mathrm{J},>=80 \text { ans et } \\
0 \text { à } 9 \% \text { de Cl.2 } \\
\text { (effectif 100) }\end{array}$ & $\begin{array}{l}\text { Cl.0 } \\
\text { Cl.1 } \\
\text { Cl.1' } \\
\text { Cl.2' } \\
\text { Cl.2" } \\
\text { Cl.+ }\end{array}$ & $\begin{array}{r}46,2 \\
60,0 \\
71,4 \\
100,0 \\
55,3 \\
50,0\end{array}$ & $\begin{array}{r}53,8 \\
40,0 \\
23,8 \\
- \\
5,3 \\
-\end{array}$ & $\begin{array}{r}- \\
4,8 \\
- \\
39,4 \\
50,0\end{array}$ \\
\hline $\begin{array}{l}\text { K. }>=80 \text { ans } \\
\text { et } 10 \text { à } 19 \% \\
\text { de Cl.2 } \\
\text { (effectif } 100)\end{array}$ & $\begin{array}{l}\mathrm{Cl} .0 \\
\mathrm{Cl} .1^{\circ} \\
\mathrm{Cl} .1^{\prime} \\
\mathrm{Cl} .2^{\prime} \\
\mathrm{Cl} .2^{\prime \prime} \\
\mathrm{Cl} .+\end{array}$ & $\begin{array}{r}76,9 \\
64,7 \\
74,1 \\
100,0 \\
63,9 \\
16,7\end{array}$ & $\begin{array}{r}23,1 \\
35,3 \\
22,2 \\
- \\
5,5 \\
-\end{array}$ & $\begin{array}{r}- \\
3,7 \\
30,6 \\
83,3\end{array}$ \\
\hline $\begin{array}{l}\text { L. }>=80 \text { ans } \\
\text { et }>=20 \% \\
\text { de Cl.2 } \\
\text { (effectif } 100 \text { ) }\end{array}$ & $\begin{array}{l}\mathrm{Cl} .0^{\prime} \\
\mathrm{Cl} .1^{\circ} \\
\mathrm{Cl} .1^{\prime} \\
\mathrm{Cl} .2^{\prime \prime} \\
\mathrm{Cl} .2^{\prime \prime} \\
\mathrm{Cl}+{ }^{+}\end{array}$ & $\begin{array}{l}81,2 \\
53,7 \\
81,8 \\
50,0 \\
72,0 \\
80,0\end{array}$ & $\begin{array}{r}18,8 \\
46,3 \\
18,2 \\
- \\
- \\
-\end{array}$ & $\begin{array}{r}- \\
- \\
50,0 \\
28,0 \\
20,0\end{array}$ \\
\hline
\end{tabular}

(") $=$ tréquence observée $=0$. 
sous-populations d'effectif inférieur ou égal à 100 (Everitt, 1977).

Au Tableau II (rubriques A et F), la fréquence observée de transition est plus grande dans le cas de la stabilisation que dans celui de l'évolution, et ce, quels que soient l'âge, l'état général ou la classe sanitaire. Tout aussi systématiquement, les classes $1^{\prime}$ et $1^{\circ}$ montrent une tendance supérieure à la dégradation, et les classes 2 ' et 2", à l'amélioration. Une évolution de l'état chlorotique du feuillage avait déjà été signalée en rapport avec des symptômes de la carence magnésienne (Weissen, 1977). Aucun individu appartenant à la classe $1^{\circ}$ en 1983 ne s'est "amélioré»; en d'autres termes, aucune cime montrant en 1983 une structure en port drapé, ne l'a perdue en 1985.

Aux rubriques $B$ et $C$, l'évolution de l'état sanitaire des arbres de classe 0 semble indépendante de l'âge : dans 7 cas sur 10 environ l'état sanitaire reste inchangé et dans les 3 restants il se dégrade et ce, quel que soit l'âge.

Les individus des autres classes sanitaires se comportent différemment : les individus de 80 ans et plus, s'ils avaient un port drapé (sans autre symptôme) en 1983 se sont plus fréquemment dégradés que les 50-80 ans et moins s'ils souffraient de chlorose légère. Affectés de légère perte d'aiguilles ou de chlorose généralisée, ils se sont plus fréquemment améliorés.

Par le biais du facteur "état sanitaire général', nous voulions observer l'eventualité d'une contagion ou d'une propagation différentielle du mal, qui se serait manifestée par une augmentation d'autant plus importante des dégradations individuelles que l'état sanitaire général du peuplement se détériorait. Les rubriques $D, E$, $\mathrm{F}$ du Tableau II ne vont pas dans ce sens si ce n'est pour la classe 2" : l'état sanitaire de ses individus s'est d'autant moins fréquemment amélioré que l'état sanitaire général des peuplements desquels ils sont issus était plus dégradé en 1983.

Les Figures 4 et 5 apportent respectivement réponse aux questions suivantes : vers quelles classes sanitaires se font préférentiellement les dégradations et améliorations ? Observe-t-on à ce sujet un comportement spécifique, pour les arbres d'âges différents ? A la Figure 4, toutes les classes sanitaires, exceptées celles de moindre effectiŕ, évoluent dans des sens divers mais avec une tendance prioritaire. Qu'il s'agisse d'amélioration ou de dégradation, cette voie privilégiée aboutit au stade chlorose.

On peut également observer qu'aucun individu "sain" ne passe dans la classe «port drapé». Nous avions déjà constaté, lors de l'analyse du Tableau II qu'aucun individu "à port drapé" ne passait dans la classe des "sains". II semblerait donc que cette structure de cime caractérise un type d'épicéa plutôt: qu'un stade de dégradation; toutefois cela reste à confirmer. Malgré la mauvaise représentativité de la classe 2', il est intéressant de noter l'unique voie d'évolution, qui mène au stade de chlorose légère, et la fréquence de transition nulle vers la dégradation. Précisons également que sur les 15 arbres de classe,+ 14 sont affectés de perte d'aiguilles sur toute la hauteur.

Sur la Figure 5 , même si les effectifs sont faibles, on remarque, à l'exception des arbres sains qui montrent un comportement équivalent quel que soit leur âge, que l'importance quantitative des transferts d'une classe à l'autre varie avec l'âge (comme observé au Tableau II), mais que les tendances d'évolution restent identiques; par exemple, les arbres âgés à port drapé qui se dégradent, sont numériquement supérieurs aux arbres plus jeunes, mais suivent les mêmes tendances. 


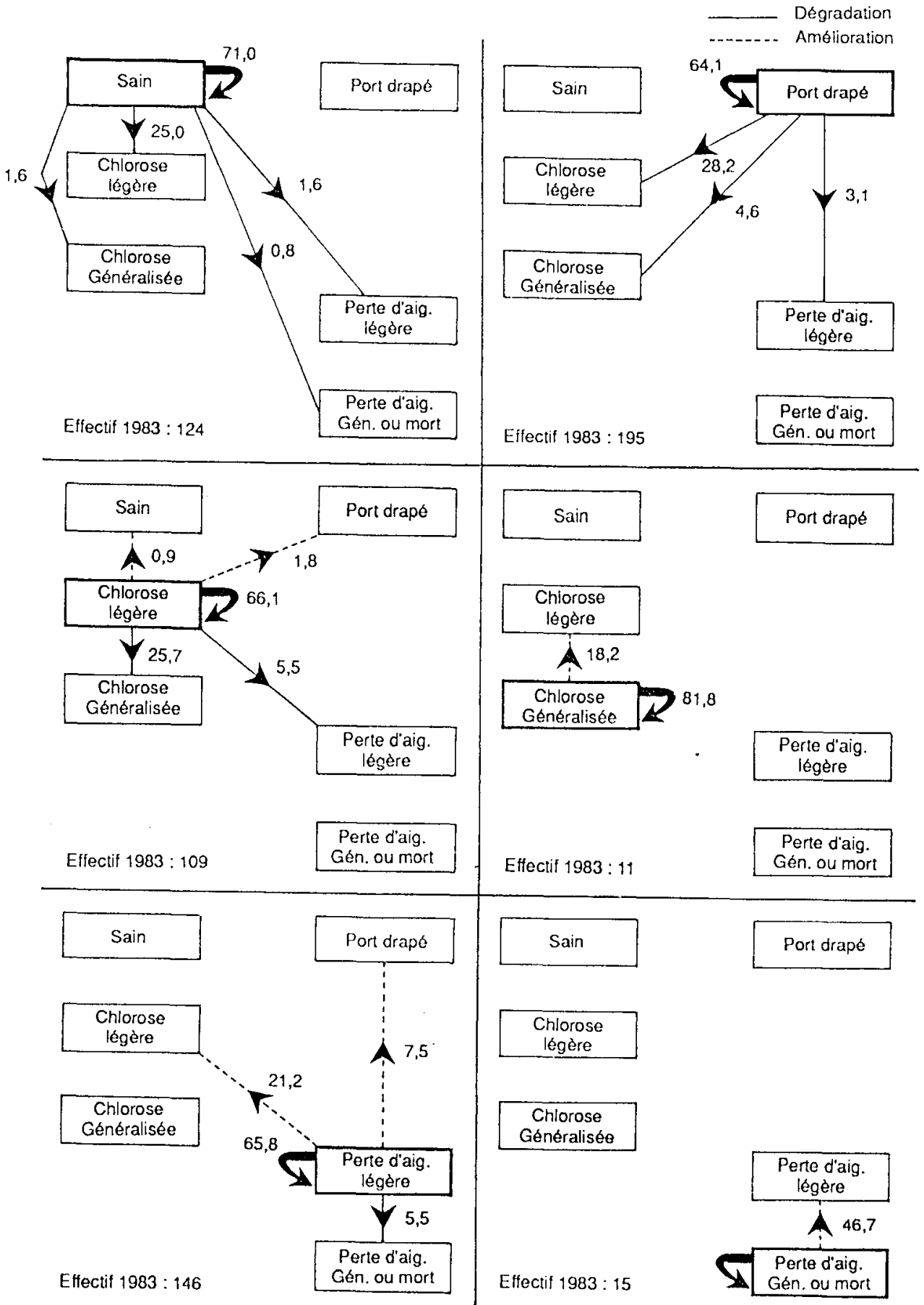

Fig. 4. Fréquence observée de transition de classe sanitaire après 2 ans (en \% des effectifs des classes sanitaires de 1983). 

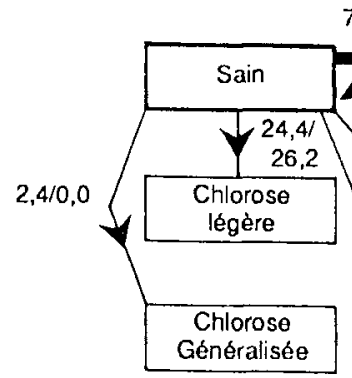

$72,0 / 69,0$
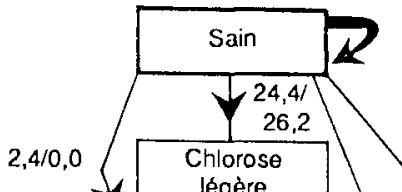
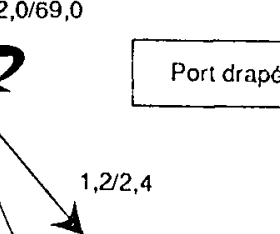

Généralisée
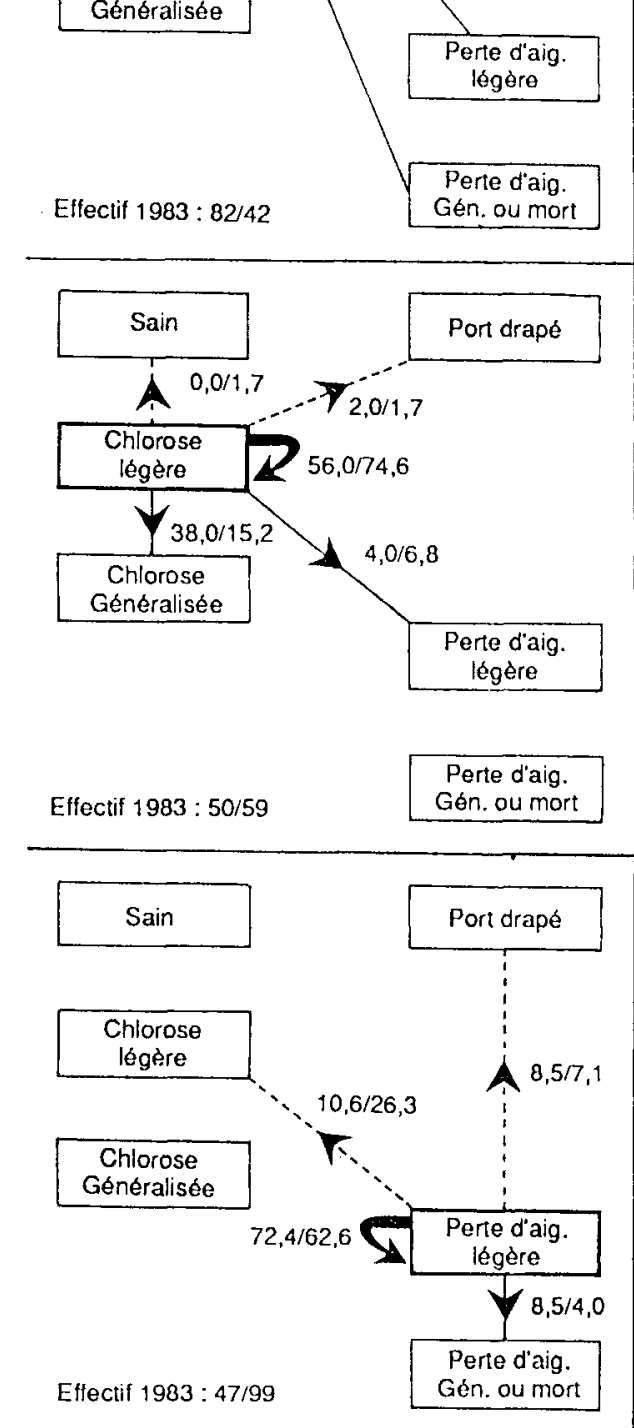

\section{$68,7 / 57,8$}
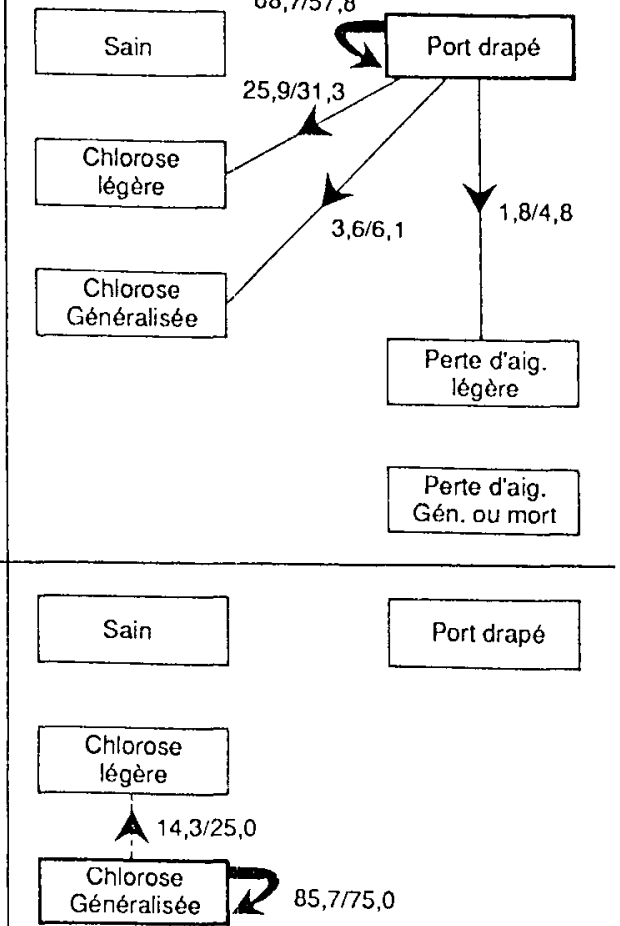

$85,7 / 75,0$

Effectif $1983: 7 / 4$

Perte d'aig.

Gén. ou mort
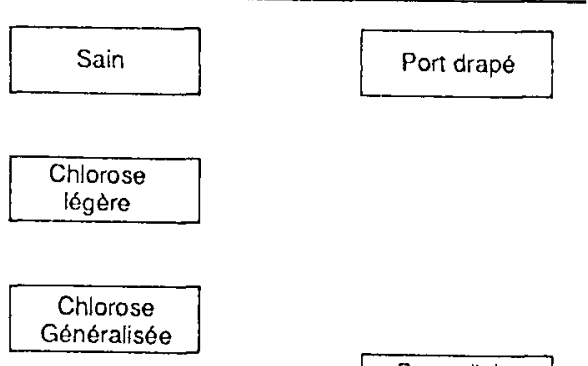

Effectif $1983: 2 / 13$

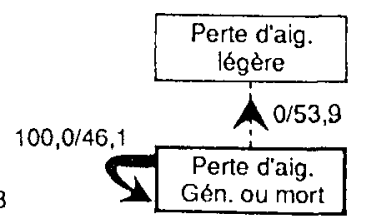

Fig. 5. Fréquence observée de transition de classe sanitaire après 2 ans avec distinction des âges : 50-79 ans / $\geq 80$ ans (en $\%$ des effectifs des classes sanitaires de 1983). 


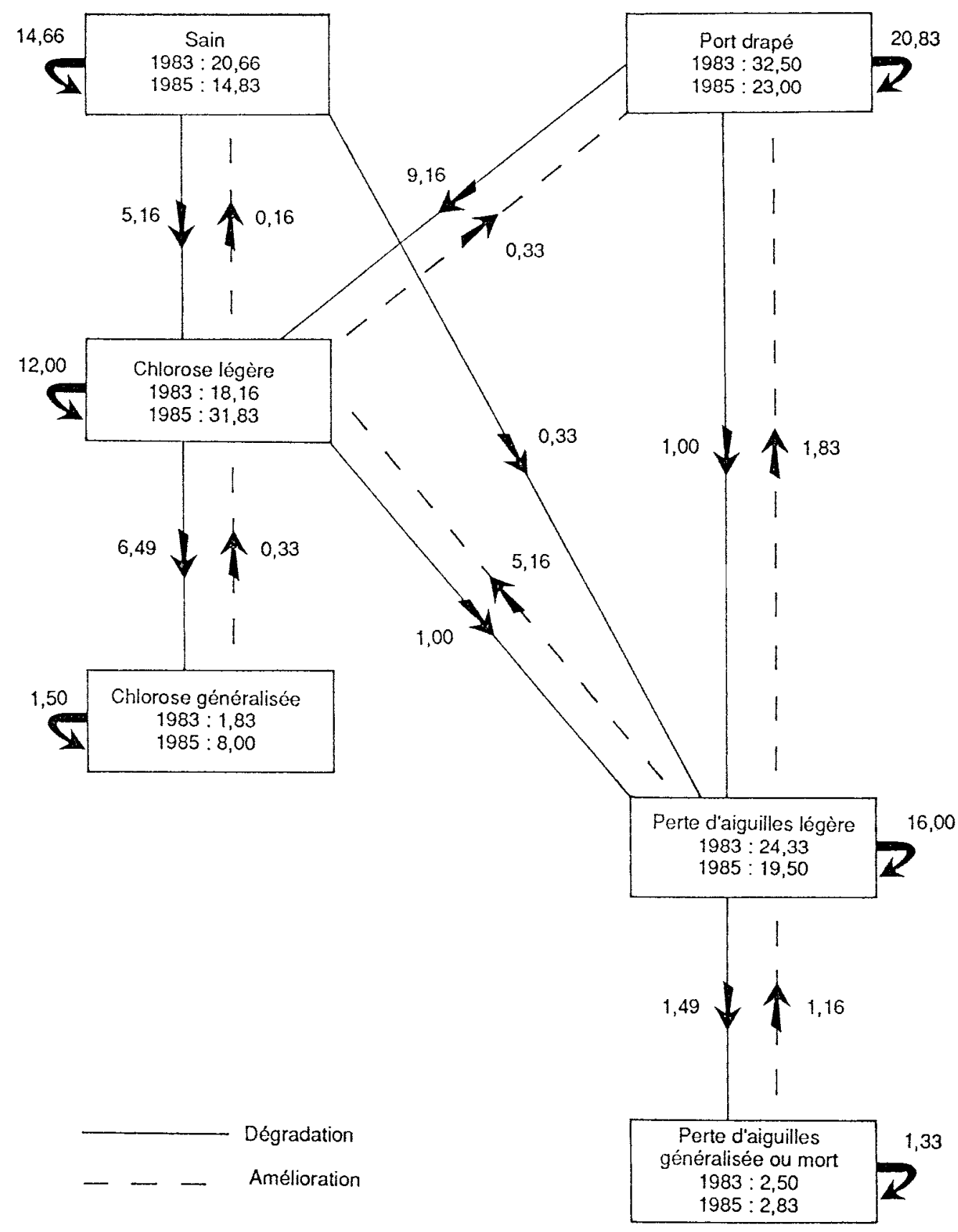

Fig. 6. Schéma évolutif de l'état sanitaire de 600 épicéas; fréquence observée de transition par classe sanitaire (en $\%$ de l'effectif total). 
Tableau III. Comparaison des effectifs observés par classe sanitaire obtenus par deux méthodes : placette permanente et inventaire systématique. EV.* : Etude de l'évolution de l'état sanitaire par placette permanente : effectif observé en 1985 (en \% de l'effectif total : 600 arbres). S. ${ }^{* *}$ : Inventaire sanitaire systématique de 1985 : effectif observé (en \% de l'effectif total : 5610 arbres).

\begin{tabular}{|c|c|c|c|c|c|}
\hline Classe & Ev. ${ }^{*}$ & & $S^{* *}$ & & Classe \\
\hline 0 & 14,83 & & & 22,50 & 0 \\
\hline 1" & 23,00 & 54,83 & & 55,20 & 1 \\
\hline $1^{\prime}$ & 31,83 & & & & \\
\hline $2^{\prime}$ & 8,00 & 27,50 & & 20,00 & 2 \\
\hline $2^{\prime \prime}$ & 19,50 & & & & \\
\hline \multirow[t]{5}{*}{+} & 2,83 & & & 0,70 & 3 \\
\hline & & & 0,70 & 0,00 & 4 \\
\hline & & & & 0,00 & 5 \\
\hline & & & & 1,50 & 6 \\
\hline & & & & & 7 \\
\hline
\end{tabular}

Les fréquences ont été exprimées jusqu'ici en pourcentage des effectifs initiaux de chaque classe. La Figure 6 utilise des pourcentages de l'effectif total et tente de montrer un schéma évolutif de l'état sanitaire de la population échantillonnée sur 2 ans. Son analyse montre l'importance quantitative du stade chlorose dans l'évolution globale du phénomène.

En comparant les pourcentages globaux par classe aux résultats issus de l'inventaire sanitaire par sondage systématique effectué en 1985 sur les mêmes zones (Tableau III) on peut apprécier la représentativité de notre échantillon qui est plutôt bonne.

\section{Commentaires}

1) L'état sanitaire des arbres observés n'a pas évolué de façon notable au cours des 2 années.

2) Sauf quelques cas particuliers, l'évolution de l'état sanitaire se manifeste de façon relativement lente.
3) Les dégradations ou améliorations se font dans des sens divers avec une tendance privilégiée vers le stade de chlorose.

II y a donc intérêt, pour toute étude relative au dépérissement, d'observer et de répertorier séparément et systématiquement les divers symptômes, avant d'avoir plus d'informations sur les causes précises du phénomène. En effet, chacun d'eux peut être indicateur d'une situation particulière (Aldinger, 1986).

L'intervalle séparant les 2 états sanitaires observés, est de 2 ans. II serait intéressant de le raccourcir de façon à pouvoir déceler la plus petite évolution possible ainsi que sa répétitivité, et détecter d'éventuelles saisons préférentielles d'évolution. A ce sujet, l'intérêt que peuvent apporter la recherche et le développement de technologies permettant la réalisation de missions aériennes ponctuelles est évident : ultraléger motorisé (Vooren et Offermans, 1985), hélicoptère télécommandé (de Henmer, 1986). Ces missions répétées permettraient de compléter nos 
observations, plus particulièrement en ce qui concerne le stade de la chlorose généralisée qui ne paraît être un passage obligé ni pour la dégradation ni pour l'amélioration.

4) La structure en port drapé ne s'est rencontrée de façon permanente qu'à partir du stade de perte d'aiguilles : aucun transfert de la classe 2" à la classe 0 n'a été observé. Néanmoins les informations manquent; il serait donc judicieux, lors de l'examen des couronnes, de préciser l'existence du port drapé quel que soit le stade de dégradation de la couronne.

5) Les fréquences observées relatives à l'amélioration de l'état sanitaire des arbres affectés de pertes d'aiguilles sont assez surprenantes; l'explication pourrait être la suivante : l'évolution dans le sens d'une amélioration se manifeste, entre autres, par l'apparition de rameaux de réaction sur la partie supérieure des branches; cette localisation contribue à donner une image plus favorable de l'état sanitaire global de la couronne sur photographie aérienne verticale et à faire passer l'arbre d'une classe 2" à $1^{\prime}$ ou même à $1^{\circ}$. En effet, les contrôles de terrain montrent que l'appréciation donnée sur base des images est exagérée par rapport au niveau de restauration réel du feuillage et qu'il y a, dans ce cas, surestimation de l'état sanitaire.

6) Le choix des facteurs "âge» et "état sanitaire général» des peuplements apparaît peu convaincant dans l'état actuel de cette première analyse; plusieurs raisons sont à invoquer. D'une part, si l'on parle d'apparition ou de disparition d'un symptôme, cela ne peut avoir de signification que si l'on en spécifie la localisation au sein de la cime. L'utilisation d'une échelle photographique de l'ordre du 1/2000 serait donc favorable à ce type d'étude ainsi qu'une précision, lors de l'examen des couronnes, quant à la présence de chlorose au stade de perte d'aiguilles.
D'autre part, l'étude a porté sur deux zones à types stationnels différents : Vielsalm et l'Hertogenwald; il s'est avéré que la zone de l'Hertogenwald est en moyenne plus dégradée mais que i'évolution vers la dégradation est proportionnellement plus importante au Grand-Bois de Vielsalm. Le facteur stationnel devrait donc à l'avenir être pris en considération parallèlement à des études de croissance et à une analyse chimique du sol et des aiguilles, pour que les critères de stratification aient plus de chance de s'exprimer.

7) II serait intéressant d'augmenter le nombre d'effectifs observés afin de rendre les tests plus puissants. D'autre part, si l'on veut généraliser les résultats à toute la zone, il faudrait se donner les moyens de choisir aléatoirement les placettes en utilisant, par exemple, un restituteur numérique; celui-ci permet par correction géométrique de la photo, de donner à chaque point image ses coordonnées géodésiques en $x, y, z$ avec une grande précision. Nous pensons en particulier à un appareil du type Stéréocord G-2 (Mohl, 1980) dont la manipulation est aisée. Dans ce cas, l'échelle du 1/5000 devrait être maintenue pour avoir suffisamment de points de calage (ITC, 1974).

8) La nature comparative ou pairée des observations paraît particulièrement intéressante et semble favorable à une diminution de la subjectivité de l'interprète, inévitable lors d'inventaire sanitaire classique au sol et dans une moindre mesure, sur photographie aérienne.

En conclusion, cette première approche laisse entrevoir des perspectives intéressantes pour l'étude générale du phénomène et des critères les plus discriminants. Une application particulièrement utile pourrait être l'analyse de l'impact des essais d'amendement calcaro-magnésien qui ont été entrepris dans nos zones test en septembre 1985. Par cette intervention, if s'agit tout autant de lutter contre 
l'acidification du milieu que de distinguer ce qui, dans la symptomatologie actuelle relève de la carence magnésienne par rapport à d'autres causes possibles, notamment de nature parasitaire (Giot et Weissen, 1985; Bonneau et Landmann, 1986). L'impact de cette fertilisation serait évalué sur la base de la méthode développée ici améliorée grâce aux enseignements qu'elle a procurés.

\section{Remerciements}

Nous tenons à remercier le ministère de la région wallonne chargé des Technologies nouvelles, grâce au financement duquel ces recherches ont pu être effectuées, ainsi que MM. Frantz Weissen et Stéphane Baeten pour leurs précieux conseils.

\section{Références}

Aboussouan N. (1985) Dépérissements de forêts en Ardenne belge et quelques facteurs de l'environnement. Mémoire de fin d'études, Université Paris Sud, Orsay

Aldinger E. (1986) Waldschäden in ungekalkten und gekalkten Fichten-Tannen-Beständen des Buntsandstein-Schwarzwaldes aus boden-und ernährungskundlicher Sicht. Premier colloque scientifique des Universités du Rhin Supérieur, recherches sur l'environnement dans la région, 27-28 juin 1986, Université Louis Pasteur et Conseil de l'Europe, Strasbourg, pp. 173-182

Bishop M.M., Fienberg S.E. \& Holland P.W. (1975) Discrete Multivariate Analysis : Theory and Practice. Mit Press, Cambridge, Massachusetts, London

Bonneau M. \& Landmann G. (1986) Essai de fertilisation. Programme DEFORPA, réunion de travail des 22, 23 et 24 janvier 1986. Centre de recherches forestières, INRA, Nancy, pp. 203207

(de) Hemmer O. (1986) Contribution à l'étude des moyens légers pour la photographie aérienne : photographie par caméras non métriques; plates-formes non conventionnelles de prise de vue. Mémoire de fin d'études, faculté des sciences agronomiques de Louvain, Université catholique de Louvain, Louvain-laNeuve

Delincé J. (1986) Aspects statistiques de l'estimation du taux de dégradation phytosanitaire sur photographies aériennes. Rapport interne, Unité de Biométrie, faculté des sciences agronomiques de l'Université catholique de Louvain, Louvain-la-Neuve.

Everitt B.S. (1977) The Analysis of Contingency Tables. Chapman and Hall, London, pp. 3966

Giot P., Maréchal P. \& Felten V. (1985) Evaluation de la télédétection comme méthode d'identification des troubles phytosanitaires en forêt. Rapport final. Convention Région Wallonne UCL, $n^{\circ} 83 / 05023$, Unité des Eaux et Forêts, faculté des sciences agronomiques de l'Université catholique de Louvain, Louvain-la-Neuve

Giot P. \& Weissen F. (1985) Développement et implications sylvicoles du dépérissement des forêts en Ardenne. Parasitica 43, 3, 105-114

Hüttl R. (1985) Neuartige Waldschäden und Nährelementversorgung von Fichtenbeständen (Picea abies Karst.) in Südwestdeutschland. Inaugural Dissertation zur Erlangung der Doktorwürde des Forstwissenschaftlichen Fakultät der Albert-Ludwigs Universität, Freiburg im Breisgau

ITC (1974) Cours d'Introduction à la Photogrammétrie. Inst. Intern. de relevés aériens et de sciences de la terre, ITC, Enschede

Mohl H. (1980) Conception and accuracy of the programm systems for the Stereocord G-2. Kongress der Internationalen Gesellschaft für photogrammetrie. Hamburg, 14, pp. 177-180

Niesslein E. \& Voss G. (1985) Was wir über das Waldsterben wissen. Deutscher InstitutsVerlag, Köln

Rehfuess K.E. (1985) Maladie de la forêt et pollution de l'air. Parasitica, 41, 3, 127-134

Remeijn J.M. (1981) Photo-interpretation in Foresty. International inst. for Aerospace survey and Earth Sciences, ITC, Enschede

Schmidt-Vogt H. (1977) Die Fichte, vol. 1, Paul Parey, Hamburg und Berlin, pp. 295-300 
Tan B.S., Chovrelat C., Bailly G. \& Bruckert S. (1986) Etude des relations entre le dépérissement et les principaux types de stations forestières du Jura Central. Programme Deforpa, réunion de travail des 22, 23 et 24 janvier 1986. Centre de recherches forestières, INRA, Nancy, 177-181

Vooren A.P. \& Offermans D.M.J. (1985) Photographie aérienne par avion ultraléger motorisé (ULM). Biotropica 17, 1, 84-88

Weissen F. (1977) Dix années d'évolution d'une deuxième génération d'épicéas fertilisés à la plantation; effets généraux des traitements. Bull. Rech. Agron. Gembloux 12, 1-2, 135-158 Weissen F. (1983) Erste Beobachtungen über die physiologische Schädigung des Nadelwaldes der belgischen Ardennen als mögliche Folge der Luftverschmutzung. Waldsterbenkonferenz, Maastricht, Eurogio Meuse-Rhin, 1114

Weissen F., Letocart M. \& Van Praag H.J. (1984) Rapport préliminaire sur les effets de la pollution atmosphérique sur les forêts de l'Ardenne. Bull. Soc. Roy. For. Belgique 91, 2, 6072 